Mathematics

\section{Classical continued fractals}

\section{from Ian Stewart}

Conirinutil) fractions are classical: they provide 'best possible' rational approximations to real numbers, expressed in the form $\alpha=1 /(a+1 /(b+1 /(c+1 / \ldots))$.$) ,$ usually written as $(a, b, c, \ldots)$ for simplicity. Their main use is in number theory. Fractals arc of much morc modern vintage. They are geometrical objects with structure on all scales. The name was coined by their inventor, Benoit Mandelbrot, to reflect their 'infinitely crinkled' shape. They are appliced to irregular behaviour and form in nature.

Can two such disparate ideas usefully be combined? Indeed they can. Jenny Harrison of the University of California at Berkeley has announced progress on a baffling problem known as the Seifert conjecture based on what she calls continued fractals. The proof is sketched in Bulletin of the American Mathematical Society 13, 147 ; 1985; full details will appear elsewhere.

The celcbrated 'hairy ball' theorem states that it is impossible to comb a hairy ball smooth. More precisely, any smooth vector field on a two-dimensional sphere must have a fixed point. In consequence, any smooth dynamical system whose state space is topologically a two-dimensional sphere must have at least one steady state. On the other hand, a hairy torus can easily be combed smooth, so dynamics on a torus can have no steady states at all. Such results have far-reaching consequences: for example they justify the choice of tori, rather than spheres, for magnetic bottles in experimental fusion reactors.

But what if the state space is a threedimensional sphere? First we must explain how such an object can be visualized. The circle, a one-dimensional sphere. can be obtained from a line by adding a "point at infinity" which is considered as lying at both ends. so that the line plus this point curls up into a circle. Similarly the 2sphere can be thought of as a plane. plus a single point at infinity. lying at both ends of every line in the plane. The horizon circle of the plane is squashed down to this single point: and much as a bag can be closed by tightening a loop around the top, the plane curls up to give a sphere. For a 3-sphere, just add a single point at infinity to three-dimensional space. To

\section{Errata}

Throughout A.M.C. Sengor's article "East Asian tectonic collage" (7 November. p. 16) the term "Indosinian" should replace "Indonesian". which was inadvertantly substituted during editing.

In Michalel Brown's article "Tectonics of metamorphism" (28 November. p.314), the wrong photomicrograph was used to illustrate Fig.2. visualize a smooth combing of the 3sphere, we imagine a family of smooth curves through 3-space, taking care that it behaves suitably 'near infinity'.

It is not hard to find ways to comb a hairy 3-sphere smoothly, with no fixed points. Other special features tend to appear, however, notably places where the hairs form a closed loop. Is there a way to comb a 3-sphere that has no fixed points and no loops? In 1950, Herbert Seifert conjectured that there is not or, more precisely, that every smooth vector field on

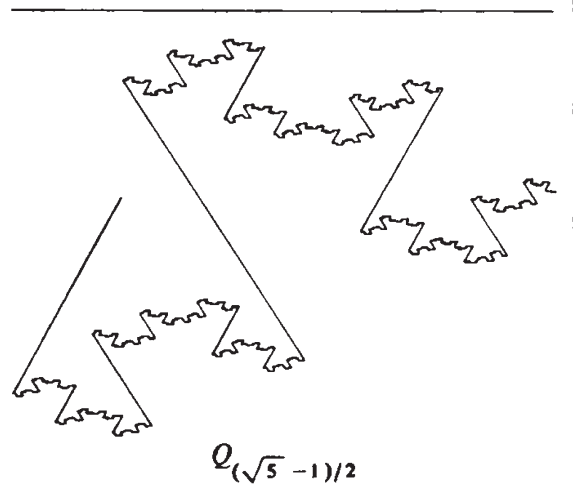

Example of a continued fractal (redrawn from Harrison, J. Bulletin of the American Mathema. tical Society 13, 147; 1985).

the 3-sphere has either a fixed point or a closed integral curve. At first, very little progress was made on this fundamental problem, because nobody knew where to start.

To describe the known results we must address the question of how smooth is "smooth"? The degree of smoothness of a curve is measured by how many times it can be differentiated. To qualify as 'smooth' it must certainly have a tangent everywhere (be once differentiable). If the position of that tangent also varies smoothly, the curve is twice differentiable, and so on. The smoothest curves are infinitely differentiable. A fancier definition lets the degree $r$ of differentiability be fractional, indeed real, as well as integral.

In 1971. Paul Schweizer (Annals Mathematica 100. 386; 1971) showed that the Seifert conjecture is false in the oncedifferentiable case. So for a degree of differentiability $r$ somewhere between 1 and $\infty$. there is a switch from false to true (unless. as may be the case, it is always false). The question is, where? In her thesis under Colin Rourke at Warwick University. Harrison showed that the switch is at a value $r>2$. Her new work. which uses similar methods, improves this to $r \propto 3$, pushing current techniques somewhere near their limit.

The starting point of her method is a deceptively simple example of discrete dynamics. Take a circle. select a real number $\alpha$ between 0 and 1 , and define a transformation $T$ of the circle by rotating it through the angle $2 \tau \alpha$. Then, under iteration of this transformation, the behaviour of points is highly sensitive to the degree of irrationality of $\alpha$. Specifically, suppose $\alpha$ has continued fraction $[a, b, c, \ldots]$, then the smaller that the entries $a, b, c, \ldots$ are. the more irrational is $\alpha$.

When the rotation $\alpha$ is rational, every point on the circle is periodic under iteration of $T$. That is, if $T$ is performed sufficiently often, every point ends up exactly where it started. If $\alpha$ becomes irrational, this periodicity starts to break up. For highly irrational $\alpha$, such as the golden number $[1,1,1,1, \ldots]$, the iterates under $T$ are evenly spread over the circle. For 'almost rational' numbers, like the Liouville number $\left[1,2, \because, 3^{3 !} \ldots\right]$, the points tend to clump together during long sequences of iteration. Thus there is a strong link between the dynamics of $T$ and the continued fraction of $\alpha$.

There are well known techniques for constructing flows on the 3-sphere from transformations such as $T$, and the central question is to determine how the degree of differentiability of the resulting flow depends on the degree of irrationality of $\alpha$. The falsity of the Seifert conjecture unless $r>2$ comes from taking $\alpha=[4,4,4, \ldots]=$ $\sqrt{ } 5-2$. Can other choices of $\alpha$ lead to smoother flows?

It is here that continued fractals enter. Harrison describes a geometric way to visualize the continued fraction of $\alpha$, by 'unfolding' the sequence of iterates of a point under the rotation $T$. This leads to a curve whose geometric structure determines arithmetical properties of the continued fraction. The curve is a fractal, and its geometric fine structure corresponds to delicate arithmetical properties, making it possible to visualize the way in which $\alpha$ is approximated by rationals, and to apply geometric reasoning. The improved value $r \geqslant 3$ arises by taking $\alpha=[N, N, N, \ldots]$ for large and even values of $N$, and by exploiting the interplay between dynamics, geometry and arithmetic. It is a vivid display of the unity of mathematics.

Meanwhile, the general Seifert conjecture remains as enigmatic as ever. Conceivably it is false even in the infinitely differentiable case. There seems little chance that current techniques can lead to a general counter-example. Harrison's methods break down for $r$ larger than 3 , leading her to suggest that it is at $r=3$ where the conjecture turns into a theorem. However, no proofs exist for any $r, \infty$ included; other considerations suggest that a proof (if indeed the conjecture is ever true) must overcome formidable technical obstacles. We may have a long wait for the complete answer.

Ian Stewart is at the Mathematics Institute. University of Warwick, Coventry CV $47 A I, U K$. 